\title{
Towards Effective Automatic Feedback for Simulator Training
}

\author{
Laura Marcano $^{1} \quad$ Anis Yazidi $^{2} \quad$ Mexhid Ferati $^{2}$ Tiina Komulainen ${ }^{1}$ \\ ${ }^{1}$ Department of Electronic Engineering, Oslo and Akershus University College of Applied Sciences (HiOA), Norway \\ ${ }^{2}$ Department of Computer Science, Oslo and Akershus University College of Applied Sciences (HiOA), Norway, \\ \{Laura.Marcano, Anis.Yazidi, Mexhid.Ferati, Tiina.Komulainen\} @hioa.no
}

\begin{abstract}
The presence of an expert instructor during simulator training is of great importance for the trainees, if not indispensable. The instructor's role is to give feedback and guide the trainees to help them make the right decisions on time as effectively as possible. The instructor starts or pauses the training scenarios when needed and facilitates reflection during and after the scenarios. However, the fact that simulator-training sessions are very dependent on the participation of a guiding instructor can be a drawback, since there are not always sufficient expert instructors to fulfil the training demands. In this work, an intelligent tutoring system (ITS) is proposed as an automatic feedback solution for simulator training. It is based on effective assessment of the system conditions using a clustering based anomaly detection technique as a core component. Furthermore, we provide insights into the design of a proper interface for our ITS. The article presents the methodology for developing such a system which consists of three stages: data collection, data analysis and delivering feedback.
\end{abstract}

Keywords: simulator training, anomaly detection, automatic feedback, intelligent tutoring system

\section{Introduction}

Operators training is a matter of great importance in different industries, as effective training leads to highly competent operators, which are able to maintain safe operations and handle abnormal plant situations when needed (Nazir et al., 2015). The most common training practice necessities the physical presence of operators in a simulator training center, where they can be in a room that is designed and furnished in such a way that it closely represents an actual control room. In there the operators interact with an Operator Training Simulator (OTS) (Kluge et al., 2009, Patle et al., 2014). During the simulator training sessions, the operators are guided by an expert instructor, and the presence of the instructor is essential since $\mathrm{s} / \mathrm{he}$ takes care of starting or pausing the simulation scenarios, gives feedback to the operators and guides them to find the best possible solution to the encountered incidents. Although the guidance of the instructors is of great advantage thanks to their experience and the knowledge they can share with the trainees, the traditional operator training practices also have several drawbacks as the operators need to be physically collocated with the instructor in the simulator training center. This scenario is illustrated in Figure 1. The mobilization of the operators can represent a high economical cost for the company investors in addition to a loss of personal time for the participants of the training session. Further, the availability of instructors is limited, which also limits the amount of operators that can be trained at the same time. Therefore, there is a need for more independent operator training practices that could overcome the current disadvantages.

In the search for more independent training practices, several simulator training sessions have been carried out with students at Oslo and Akershus University College of Applied Sciences (HiOA), in order to observe and evaluate the needs of the trainees during simulator training sessions (Marcano and Komulainen, 2016, Marcano et al., 2017b). In our latest research (Marcano et al., 2017a) an automatic assessment tool was tested, and even though the trainees found it helpful, they still considered that the feedback and direction given by the instructor was necessary. Hitherto, this indicated that the relevance of the instructor lies in the feedback and

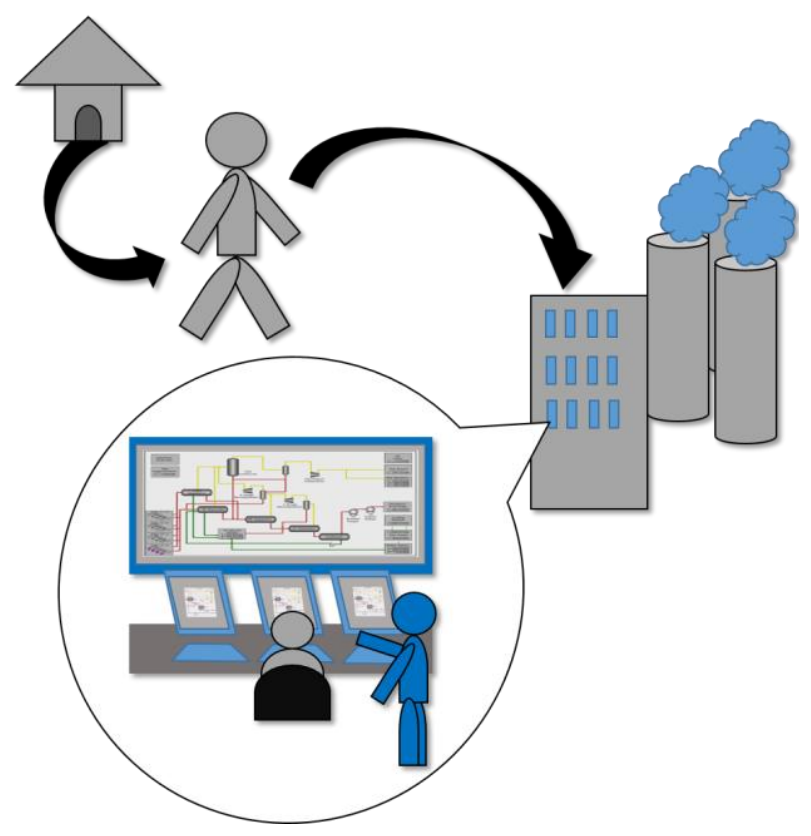

Figure 1. Traditional operator training 
guidance s/he gives to the trainees. Consequently, for an independent simulator training session to be successful, an effective automatic feedback is required. In this way, the dependence on the instructor can be decreased for certain aspects of the training scenarios. Moreover, there already exists extensive research on systems that represent an alternative to expert human instructors and provide automatic guidance and feedback to trainees. These systems are known as intelligent tutoring systems (ITS) (Gonzalez-Sanchez et al., 2014). ITS are better known for being implemented in academic purposes, especially in higher education. Nevertheless, the concept of ITS is widely applied in different fields as it consists of offering independent computerized learning, which in the current era could be applicable to almost everything. A relevant example of ITS implementation is found in game-based training (Goldberg and CannonBowers, 2015, Hooshyar et al., 2016), which can be compared to simulator training, and is a motivation for considering the implementation of ITS as a possible option towards more independent simulator training practices.

Combining an ITS with simulator training implies that the ITS must know the state of the process in order to be able to give any feedback to the user. It must be able to recognize the abnormal situations when they occur and inform the trainee about it in an effective way, so that $\mathrm{s} / \mathrm{he}$ can handle the emergencies in time and prevent them from escalating.

There are different key parameters that characterize industrial processes, such as temperature, pressure, flow rates, energy consumption, mass and energy balances, environmental factors, equipment key performance indicators (KPI) and optimal value ranges for some key measurements of the process. Prompt feedback from the ITS can be based on the analysis of these key parameters, which can represent an extensive amount of data. Therefore, the ITS integrated to simulator training must be based on a proper data analysis technique. A well-known technique for monitoring the wellfunctioning of a system based on data flow is anomaly detection. In simple terms, anomaly detection aims to identify patterns in data that move away from the expected behavior. Anomaly detection is applied within an extensive range of different domains such as fraud detection for credit cards, health care, intrusion detection for cyber-security, and fault detection in safety critical systems (Chandola et al., 2009). The industrial processes simulated for operator training are safety critical systems that produce a large amount of data. Due to this reason, anomaly detection is considered as a suitable technique for the ITS to determine the process status. This work proposes a suitable architecture design for an ITS based on anomaly detection, integrated into an operator training simulator. The aim of this proposal is to settle a basis towards autonomous simulator training.

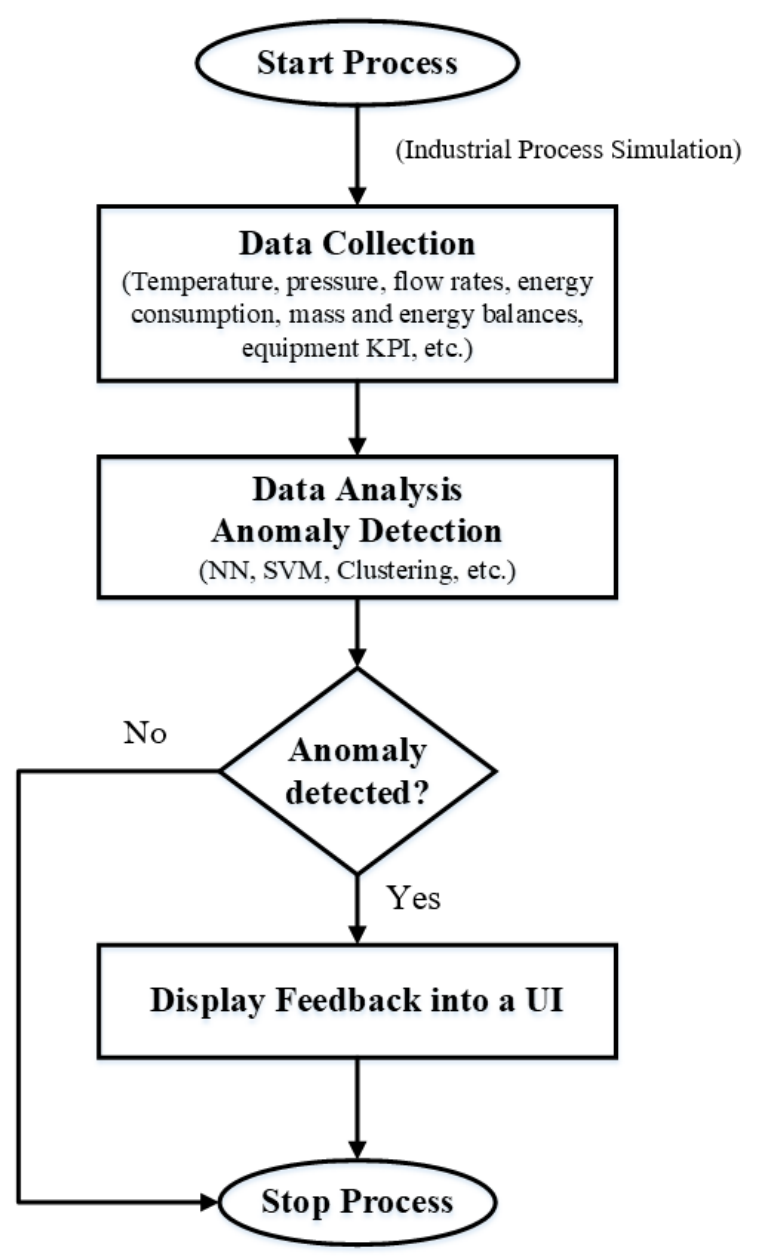

Figure 2. Technical Implementation

\section{Methodology}

The functioning of the proposed ITS integrated to a dynamic simulator consists of three stages online. First, data from the simulation process is collected. Next, the data must be analyzed in order to determine the status of the process and identify changes or abnormalities. Finally, the results from the data analysis are shown to the trainee through a smart user interface (UI), so that they can easily understand the status of the process. Further, the presentation of the process status should be accompanied with relevant feedback and suggestions when necessary to improve the system conditions. Figure 2 shows an overview of the technical implementation of the proposed ITS for simulator training.

\subsection{Data collection}

Relevant information that describe the system must be collected. Industrial processes are monitored by distributed control systems, which are characterized by producing a large amount of data that correspond to 


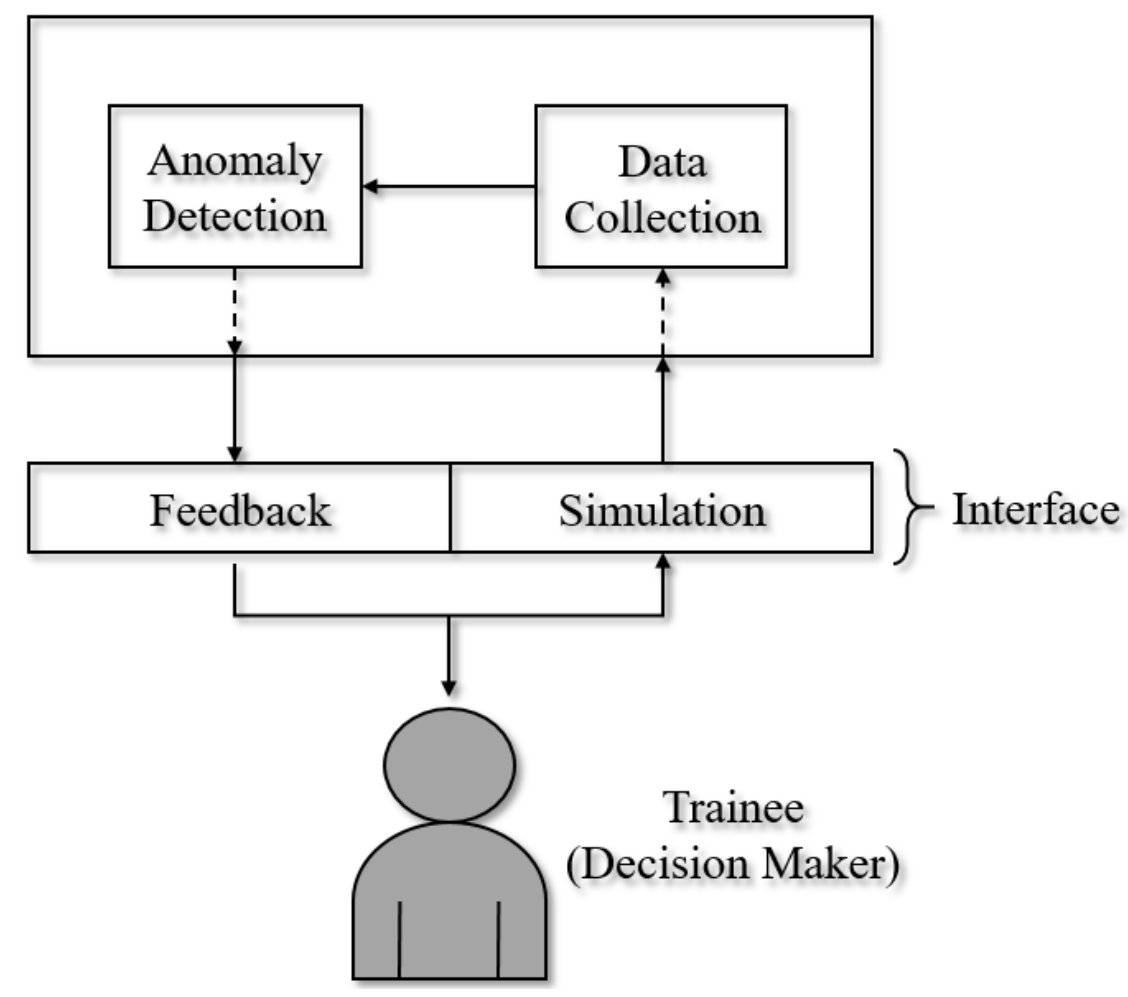

Figure 3. Interaction design

many different sensor signals with information about the process. Therefore, the data should be chosen strategically, it must be determined which parameters are significant enough to represent the status of the system. Further, since industrial processes consist of a combination of different smaller subsystems, in order to be able to monitor the process to all its extension, relevant data must be gathered from each of the subsystems involved in the process. Some examples of pertinent parameters that describe an industrial process are temperature, pressure, flow rates, energy consumption, mass and energy balances, environmental factors, and KPI of the equipment. Further, there exist optimal value ranges for certain key measurements of the process.

In order to handle data properly, a well-structured database is needed. As long as the user is training with the simulator, the representative parameters of the process should be sent to the database, so that the status of the process is analyzed continuously. This is illustrated in Figure 3, which was inspired by Figure 1.1 shown in Chapter 1 of Polson and Richardson (2013).

\subsection{Data Analysis}

Data handling is an important aspect considering that numerous processes generate large amount of data, and it is imperative that this data is properly studied and analyzed so these processes can be kept with a good functioning. Anomaly detection consists of finding patterns in data that do not follow the expected behavior
(Chandola et al., 2009). Any deviation from the normal profile of the model is considered as an anomaly (Zaher and McArthur, 2007); its most common applications are related to cyber security. However, the concept of anomaly detection is applied in different fields, among which is the industrial damage detection, which refers to detection of different faults and failures in complex industrial systems (Chandola et al., 2009). This highlights the reason why this technique is considered suitable for monitoring the status of the simulation processes during simulator training.

On the other hand, simulator training is commonly implemented to train operators working with industrial processes; these processes are organized as distributed systems. A distributed anomaly detection technique is needed, so that a proper monitoring of an entire industrial process can be done, considering that relevant changes or abnormalities in the process can come from many different sources. Centralized methods are not suitable for distributed systems since they may not scale easily for real-time management (Thottan and Chuanyi, 1998). In order to detect anomalies for a complex system on a global level, the integration of information about the anomalies identified in single locations is required (Banerjee et al., n.d.). This means that an entity capable of integrating multiple data sources and interpretation techniques is needed.

There exists different anomaly detection techniques. In a survey conducted by Chandola et al. (2009) these techniques are organized into four main categories: 


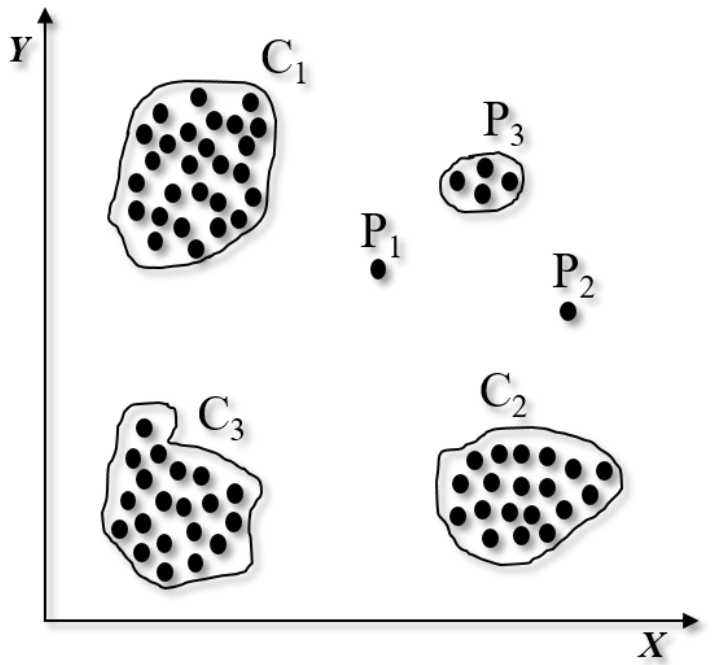

Figure 4. Example of clustering based outlier detection

- classification based, among which are included neural networks (NN), Bayesian network, support vector machine (SVM) and rule based;

- nearest neighbor based, which includes density and distance based techniques;

- clustering based;

- and statistical, which can be parametric or nonparametric.

For the case of the system proposed in this work, the chosen anomaly detection technique is clustering based, due to its simplicity and practicality for evaluating changes in data and sequences.

Clustering is used to group similar data instances into clusters (Chandola et al., 2009). Based on this concept, "normal" data instances belong to a specific cluster, while anomalies do not belong to any cluster.

For the proposed ITS, the first step is to observe the key parameters received from the simulated industrial process over a time window. The average of these parameters characterizes the "normal" system behavior. After gathering enough data to describe the system's normal behavior, the clusters can be created. Consequently, abnormal operating modes can be identified if data falls out of the defined clusters. Figure 4 shows a simple example of this, where $\mathrm{C}_{1}, \mathrm{C}_{2}$ and $\mathrm{C}_{3}$ are regions of normal behavior, while $\mathrm{P}_{1}, \mathrm{P}_{2}$ and $\mathrm{P}_{3}$ are outliers that fall out of the defined clusters.

Moreover, the dynamicity of the system can be learned by studying the transitions that occur among clusters, the system can move from a normal behavior to an abnormal behavior. Nevertheless, the transitions can also indicate that the system is moving from a normal behavior to another type of normal behavior. Hence the importance of studying the dynamicity, so normal and abnormal changes are identified and classified correctly. The evaluation of the transitions among clusters is known as sequential pattern mining
(Rahman et al., 2016). Based on the definition given in Rahman et al. (2016), a sequence of cluster transitions can be defined as follows:

Let $C=\left\{c_{1}, c_{2}, \ldots, c_{l}\right\}$ be a set of all clusters. A cluster set $C_{x}=\left\{c_{1}, c_{2}, \ldots, c_{m}\right\} \subseteq C$ is a nonempty and unordered set of distinct clusters. A sequence $S$ is an ordered list of clusters expressed as $\left\langle C_{1}, C_{2}, C_{3}, \ldots, C_{n}\right\rangle$ such that $C_{k} \subseteq C(1 \leq k \leq n)$. An example of a sequence can be $S_{1}=\langle\{3\},\{1\},\{2\},\{1,3\}\rangle$, which is shown in Figure 5. Based on the normal sequence patterns of the system, abnormal changes can be identified to inform the operator.

\subsection{Delivering Feedback}

Once the data is analyzed, the results of the analysis must be presented to the user. This is a crucial stage, because successful training is related to effective feedback. Consequently, it is necessary to design a suitable user interface, which shows effectively what is relevant for the user to know, in a clear and understandable way. Figure 3 shows the design of the interaction between the user and the interface of the proposed ITS. The trainee should be able to see the simulation and the feedback about the process status at the same time. As long as the simulation is active, data should be gathered and analyzed in order to identify if there is any abnormal change in the process. Any abnormality must be indicated to the trainee together with some guidance on what could have caused the situation or how it could be solved. However, the trainee has the final decision on how to proceed after getting feedback from the system.

The design of the user interface is a very important matter because it must guarantee that the user will be comfortable with it, it should be easy to use and understand, and it should enable the users to attain the

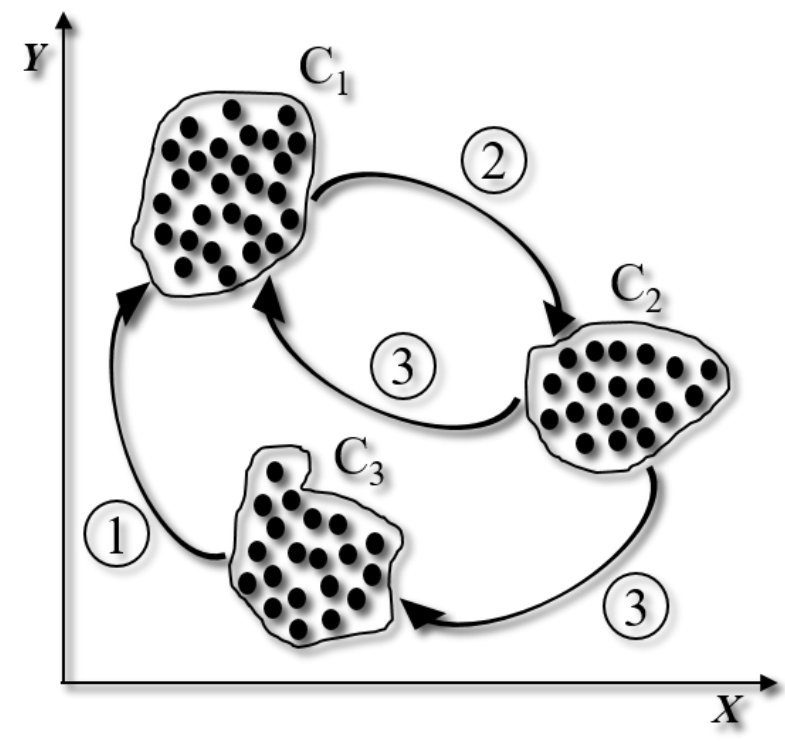

Figure 5. Example of a sequence of clusters transition 


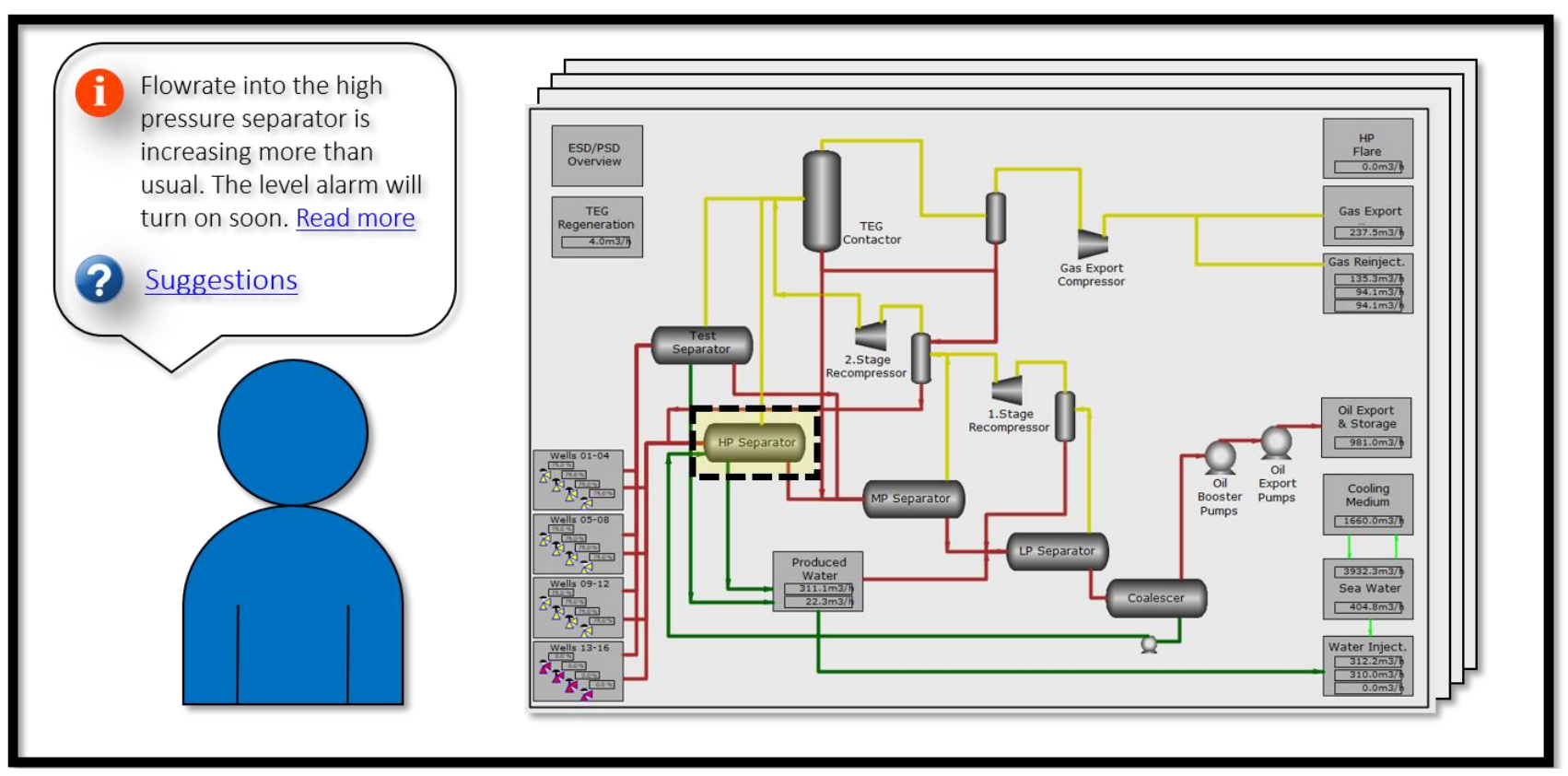

Figure 6. Example of a user interface for the proposed ITS (K-Spice®)

goal of handling successfully any abnormality or emergency that may occur in the system. Stone et al. (2005) indicate that a good user interface design promotes easy, natural, and engaging interaction between the user and the system, making the user forget that $\mathrm{s} / \mathrm{he}$ is using a computer. These characteristics must (Chandola et al., 2009) be targeted in order to ensure a proper feedback delivery to the user about the simulation process.

There exists different guidelines to create a proper interface, and according to Johnson (2010) the two bestknown are Shneiderman and Plaisant (2009) and Nielsen and Molich (1990). There are several matching points between these two guidelines, and both agree that a user interface must (Nielsen, 1995):

- be consistent: the user should not be wondering about the meaning of what is shown on the interface i.e., it should follow platform conventions.

- prevent errors: the design should be made in such a way that it avoids the user from making mistakes.

- make the users feel they are in control: one way to give freedom to the user is by supporting undo and redo options.

- minimize the user's memory load: the user should not have to remember information from one dialogue to another, it should be easy to access any information needed.

Based on these guidelines, Figure 6 illustrates how the final user interface of the proposed ITS should look like. The figure shows an example where the tutoring system informs the trainee that the flowrate into the highpressure separator is increasing more than usual and that the alarm will activate soon. It also offers more information in case the trainee wants to know more details about what is happening in the system. Further, the tutoring system generates suggestions to help the user handle the abnormal situation, enabling her/him to decide freely if s/he wants to read the suggestions or not.

\section{Discussion}

It is expected that the implementation of automatic feedback for simulator training will lead to more independent operators and will enable the possibility of training a higher number of operators at the same time, currently an instructor may work with two to six operators in one simulation session. With an intelligent tutoring system, this number can increase. Moreover, given the independence offered by an ITS, operators will be able to train and practice with the simulator in any place, at any time, which will allow them to be more prepared for the training at the simulator training center. Hence, the training time at the center can be decreased, which in turn represents economic savings for the company investors and time saving for the operators and the instructors.

With the ITS, the operators will be able to receive feedback shortly after they make changes in the process or while they are following certain procedures, which will give them the opportunity to solve in time any mistake they could possibly make. Further, the ITS, compared to control alarms, is more susceptible to process changes, which will also help the operators to practice identifying and solving problems before they escalate. It is expected that all the proposed advantages of the ITS will give as a result more prepared and competent operators. 


\section{Conclusion and Future Work}

In this article, the integration of an intelligent tutoring system (ITS) into simulator training was proposed. This, with the aim to offer training independence to industrial operators through an effective automatic feedback tool. There exist important challenges with this proposal since in order to produce effective feedback for the operator, the status of the process must be evaluated constantly. Because of that, a clustering based anomaly detection technique is suggested. Further, the way feedback is delivered to the operator is an important challenge, because if relevant information fails to be delivered effectively, the operator will not be able to understand the message given by the ITS. Therefore, guidelines for user interface design especially for simulation and training contexts should be studied.

Future work includes the development and actual implementation of the proposed ITS. The ITS must be tested by users during simulator training sessions. A careful study of user's experience about the tool must be done in order to evaluate the performance of the ITS and its usability, so that it can be improved and developed further.

\section{Acknowledgements}

The authors would like to thank Marielviz Canelones and the $\mathrm{PhD}$ Candidate Ashish Rauniyar for their valuable input in designing Figures 1 and 2, respectively.

\section{References}

Banerjee, A., Chandola, V., Kumar, V., Srivastava, J. \& Lazarevic, A. n.d. Anomaly Detection: A Tutorial [Online]. University of Minnesota and United Technology Research Center. Available: https://www.siam.org/meetings/sdm08/TS2.ppt [Accessed 21 April 2017].

Chandola, V., Banerjee, A. \& Kumar, V. 2009. Anomaly detection: A survey. ACM Computing Surveys (CSUR), 41, 1-58. DOI 10.1145/1541880.1541882

Goldberg, B. \& Cannon-Bowers, J. 2015. Feedback source modality effects on training outcomes in a serious game: Pedagogical agents make a difference. Computers in Human Behavior, 52, 1-11. http://doi.org/10.1016/j.chb.2015.05.008

Gonzalez-Sanchez, J., Chavez-Echeagaray, M. E., Vanlehn, K., Burleson, W., Girard, S., Hidalgo-Pontet, Y. \& Zhang, L. 2014. A system architecture for affective meta intelligent tutoring systems. DOI 10.1007/978-3-319-07221-0_67
Hooshyar, D., Ahmad, R. B., Yousefi, M., Fathi, M., Horng, S.-J. \& Lim, H. 2016. Applying an online game-based formative assessment in a flowchart-based intelligent tutoring system for improving problem-solving skills. Computers \& Education, 94, 18-36. https://doi.org/10.1016/j.compedu.2015.10.013

Johnson, J. 2010. Introduction. Designing with the Mind in Mind. Boston: Morgan Kaufmann. https://doi.org/10.1016/B978-0-12-375030-3.00020-X

Kluge, A., Sauer, J., Schüler, K. \& Burkolter, D. 2009. Designing training for process control simulators: a review of empirical findings and current practices. Theoretical Issues in Ergonomics Science, 10, 489-509. DOI 10.1080/14639220902982192

Marcano, L. \& Komulainen, T. 2016. Constructive Assessment Method for Simulator Training. $9^{\text {th }}$ EUROSIM Congress on Modelling and Simulation. Oulu, Finland: IEEE. DOI 10.1109/EUROSIM.2016.190

Marcano, L., Komulainen, T. \& Haugen, F. A. 2017a. Implementation of performance indicators for automatic assessment. $27^{\text {th }}$ European Symposium on Computer Aided Process Engineering - ESCAPE 27. Barcelona, Spain: (Accepted on May 2017).

Marcano, L. A., Komulainen, T. M. \& Sannerud, R. 2017b. An Interactive Teaching Module for Combined Simulation and Laboratory Work. MNT-konferansen 2017. Oslo, Norway.

Nazir, S., Sorensen, L. J., Øvergård, K. I. \& Manca, D. 2015. Impact of training methods on Distributed Situation Awareness of industrial operators. Safety Science, 73, 136145. http://dx.doi.org/10.1016/i.ssci.2014.11.015

Nielsen, J. 1995. 10 Usability Heuristics for User Interface Design [Online]. Available: https://www.nngroup.com/articles/ten-usability-heuristics/ [Accessed 22 May 2017].

Patle, D. S., Ahmad, Z. \& Rangaiah, G. P. 2014. Operator training simulators in the chemical industry: review, issues, and future directions. Reviews in Chemical Engineering, 30, 199-216. DOI 10.1515/revce-2013-0027

Polson, M. C. \& Richardson, J. J. 2013. Foundations of Intelligent Tutoring Systems, Routledge. doi:10.4324/9780203761557. 978-1-138-16101-6

Rahman, A., Xu, Y., Radke, K. \& Foo, E. 2016. Finding anomalies in SCADA logs using rare sequential pattern mining. DOI 10.1007/978-3-319-46298-1_32

Stone, D., Jarrett, C., Woodroffe, M. \& Minocha, S. 2005. User interface design and evaluation, Amsterdam, Elsevier. ISBN: 978-0-12-088436-0

Thottan, M. \& Chuanyi, J. 1998. Proactive anomaly detection using distributed intelligent agents. IEEE Network, 12, 2127. DOI $10.1109 / 65.730748$

Zaher, A. S. a. E. \& Mcarthur, S. D. J. 2007. A multi-agent fault detection system for wind turbine defect recognition and diagnosis. 2007 IEEE Lausanne Powertech Proceedings. IEEE. DOI 10.1109/PCT.2007.4538286 